\title{
Cinnamaldehyde protects against oxidative stress and inhibits the TNF- $\alpha$-induced inflammatory response in human umbilical vein endothelial cells
}

\author{
NAM-YI KIM ${ }^{1}$, NGUYET-TRAN TRINH ${ }^{1}$, SANG-GUN AHN ${ }^{2}$ and SOO-A KIM ${ }^{1}$ \\ ${ }^{1}$ Department of Biochemistry, Dongguk University College of Oriental Medicine, Gyeongju, North Gyeongsang 38066; \\ ${ }^{2}$ Department of Pathology, Chosun University College of Dentistry, Gwangju 61452, Republic of Korea
}

Received November 26, 2019; Accepted April 3, 2020

DOI: $10.3892 / \mathrm{ijmm} .2020 .4582$

\begin{abstract}
Oxidative stress and inflammation play critical roles in the development of cardiovascular diseases. Cinnamaldehyde (CA) is a natural compound from Cinnamomum cassia, and its anticancer, antimicrobial and anti-inflammatory activities have been widely investigated. In the present study, the cytoprotective and anti-inflammatory effects of CA on $\mathrm{H}_{2} \mathrm{O}_{2^{-}}$or tumor necrosis factor (TNF)- $\alpha$-exposed human umbilical vein endothelial cells (HUVECs) were examined. $\mathrm{CA}$ and its natural derivative, 2-methoxycinnamaldehyde (MCA), markedly increased the cellular protein level of heme oxygenase-1 (HO-1) and promoted the translocation of nuclear factor erythroid 2-related factor 2 (Nrf2) to the nucleus. CA-mediated Nrf2/HO-1 activation protected the HUVECs from $\mathrm{H}_{2} \mathrm{O}_{2}$-induced oxidative stress, which promotes apoptosis. HO-1 depletion by siRNA attenuated the CA-mediated cell protective effects against oxidative stress. Additionally, CA markedly inhibited the adhesion of U937 monocytic cells to HUVECs by decreasing the expression level of vascular cell adhesion protein 1 (VCAM-1). An in vivo experiment confirmed the anti-inflammatory effects of CA, as lipopolysaccharide (LPS)-induced inflammatory cell infiltration was effectively inhibited by the compound. Overall, these observations suggest that $\mathrm{CA}$ may be used as a therapeutic agent for oxidative stress-mediated cardiovascular diseases, such as atherosclerosis.
\end{abstract}

Correspondence to: Professor Soo-A Kim, Department of Biochemistry, Dongguk University College of Oriental Medicine, 123 Dongdae-ro, Gyeongju, North Gyeongsang 38066, Republic of Korea

E-mail:ksooa@dongguk.ac.kr

Key words: atherosclerosis, human vascular endothelial cell, cinnamaldehyde, oxidative stress, tumor necrosis factor- $\alpha$, heme oxygenase-1, nuclear factor erythroid 2-related factor 2

\section{Introduction}

Oxidative stress produces excessive reactive oxygen species (ROS), such as hydrogen peroxide $\left(\mathrm{H}_{2} \mathrm{O}_{2}\right)$, superoxide radical $\left(\mathrm{O}_{2}^{-}\right)$and hydroxyl radical ('OH). The insufficient elimination of ROS by antioxidant mechanisms perturbs cellular redox homeostasis (1-3). Previous studies have demonstrated that excessive ROS generation leads to the peroxidation of membrane lipids, disturbs cellular antioxidant signaling and induces apoptosis (4). ROS also play critical roles as pathogenic factors in the development of cardiovascular disease by promoting the apoptosis of endothelial cells $(5,6)$. Therefore, protecting endothelial cells from apoptosis induced by oxidative stress appears to be an effective strategy for the prevention and treatment of cardiovascular disease.

Cinnamaldehyde (CA) is a major compound in cinnamon obtained from the stem bark of Cinnamomum cassia (7). CA exhibits various biological properties, such as antibacterial, anti-inflammatory and anticancer activities (8-10). Additionally, Song et al reported the antioxidative and anti-inflammatory properties of CA in a model of acute myocardial ischemia (11). However, the precise molecular mechanisms responsible for the protective effects of CA on cells remain to be elucidated.

Atherosclerosis and its complications are the most common causes of mortality in humans, and oxidative stress and inflammation play critical roles in the development of cardiovascular diseases. During the complex developmental process of atherosclerosis, damage to vascular endothelial cells is considered an initiator of vascular disease (12-14). The authors have recently reported that $\mathrm{CA}$ actively protects human dental pulp cells (hDPCs) under oxidative stress conditions by inducing nuclear factor erythroid 2-related factor 2 (Nrf2) activation and subsequent heme oxygenase-1 (HO-1) expression (15). As a transcription factor, Nrf 2 regulates the expression of antioxidant proteins that protect cells against oxidative stress. Under conditions of stress, $\mathrm{Nrf} 2$ is activated and translocated from the cytoplasm to the nucleus where it induces the expression of antioxidant defense enzymes, such as HO-1 $(16,17)$. CA also exerts protective effects against the oxidized low-density lipoprotein (ox-LDL)-induced proliferation and migration of vascular smooth muscle cells (VSMCs) (18). Additionally, 2-methoxycinnamaldehyde (MCA), a natural derivative of 
CA, has been shown to exhibit anti-atherosclerotic activity by inhibiting the proliferation and migration of human aortic smooth muscle cells (HASMCs) (19). These findings strongly suggest that $\mathrm{CA}$ is a candidate against for the treatment of cardiovascular diseases. However, to the best of our knowledge, no available studies to date have described the effects of CA on vascular endothelial cells exposed to oxidative stress.

In the present study, the value of $\mathrm{CA}$ as an anti-atherosclerotic agent was assessed. The cytoprotective effects of $\mathrm{CA}$ and the underlying molecular mechanisms in $\mathrm{H}_{2} \mathrm{O}_{2}$-exposed human umbilical vein endothelial cells (HUVECs) were examined. In addition, the anti-inflammatory effects of CA in HUVECs and Sprague-Dawley rats were investigated.

\section{Materials and methods}

Materials. CA, zinc protoporphyrin (ZnPP) and SB202190 were obtained from Sigma-Aldrich; Merck KgaA. MCA and 2'-hydroxycinnamaldehyde (HCA) were purchased from Santa Cruz Biotechnology, Inc., and $\mathrm{H}_{2} \mathrm{O}_{2}$ was supplied by CalBiochem. Cobalt protoporphyrin (CoPP) was purchased from MP Biomedicals and calcein AM was supplied by Invitrogen; Thermo Fisher Scientific, Inc. Recombinant human tumor necrosis factor (TNF)- $\alpha$ was obtained from R\&D Systems, Inc.

Cell culture and viability assay. HUVECs (ATCC, CRL-1730) were maintained at $37^{\circ} \mathrm{C}$ under $5 \% \mathrm{CO}_{2}$ in endothelial cell medium (ECM) supplemented with reagents provided in a complete kit (ScienCell Research Laboratories, Inc.). Human monocytic U937 cells (ATCC, CRL-1593) were maintained in RPMI-1640 medium supplemented with $10 \%$ FCS, 100 units $/ \mathrm{ml}$ penicillin and $100 \mu \mathrm{g} / \mathrm{ml}$ streptomycin. For viability assays, HUVECs were seeded in 12-well plates at a density of $5 \times 10^{4}$ cells $/ \mathrm{ml}$, cultured overnight and treated with various concentrations of CA, MCA or HCA for $24 \mathrm{~h}$. Cell viability was measured using a 3-(4,5-dimethylthiazol2-yl)-2,5-diphenyltetrazolium bromide (MTT) assay according to a previously described method (20).

Cytotoxicity assay. Cell cytotoxicity was determined by measuring lactate dehydrogenase (LDH) activity following treatment with CA, MCA or HCA using a CytoTox $96^{\circledR}$ nonradioactive assay kit (Promega Corporation) according to the manufacturer's instructions.

Western blot analysis. HUVECs were treated with various concentrations of CA or MCA for various periods of time. The cells were treated with SB202190 $(20 \mu \mathrm{M}) 2 \mathrm{~h}$ prior to CA treatment. The cells were washed twice with cold PBS and lysed in RIPA buffer (PBS supplemented with $1 \%$ NP-40, $0.5 \%$ sodium deoxycholate, $1 \mathrm{mM}$ phenylmethylsulfonyl fluoride, $1 \mu \mathrm{g} / \mathrm{ml}$ aprotinin and $1 \mathrm{mM}$ sodium orthovanadate). The cell lysates were incubated at $4^{\circ} \mathrm{C}$ for $30 \mathrm{~min}$ and then cleared by centrifugation at $10,000 \mathrm{x}$ g for $10 \mathrm{~min}$. Alternatively, cytoplasmic and nuclear fractions were obtained using a previously described subcellular fractionation method (21). The protein concentration was determined using Protein Assay Dye Reagent Concentrate (Bio-Rad Laboratories, Inc.). A total of $10 \mu \mathrm{g}$ of proteins were resolved on 10 or $12 \%$ SDS-PAGE and then transferred to a nitrocellulose membrane. The membrane was blocked in Tris-buffered saline (TBS) containing 5\% non-fat milk for $4 \mathrm{~h}$ at room temperature. Immunoblotting was performed overnight at $4^{\circ} \mathrm{C}$ using the following antibodies: Anti-HO-1 (1:1,000; cat. no. ADI-SPA-896; Enzo Life Sciences, Inc.), anti-AKT (1:2,000; cat. no. 4691s; Cell Signaling Technology, Inc.), anti-p-AKT (1:2,000; cat. no. 4060s; Cell Signaling Technology, Inc.), anti-ERK (1:2,000; cat. no. 4695s; Cell Signaling Technology, Inc.), anti-p-ERK (1:2,000; cat. no. 4370s; Cell Signaling Technology, Inc.), anti-p38 (1:2,000; cat. no. 8690s; Cell Signaling Technology, Inc.), anti-p-p38 (1:2,000; cat. no. 4511s; Cell Signaling Technology, Inc.), anti-p-nuclear factor (NF)- $\kappa \mathrm{B}(1: 2,000$; cat. no. 3033s; Cell Signaling Technology, Inc.), anti-poly(ADP-ribose) polymerase (PARP) (1:2,000; cat. no. 9542s; Cell Signaling Technology, Inc.), anti-cleaved-PARP (1:2,000; cat. no. 5625s; Cell Signaling Technology, Inc.), anti-Nrf2 (1:1,000; cat. no. sc365949; Santa Cruz Biotechnology, Inc.), anti-NF-кB (1:2,000; cat. no. sc8008; Santa Cruz Biotechnology, Inc.), anti-intercellular adhesion molecule 1 (ICAM-1) (1:2,000; cat. no. sc8439; Santa Cruz Biotechnology, Inc.), anti-vascular cell adhesion protein 1 (VCAM-1) (1:2,000; cat. no. sc8304; Santa Cruz Biotechnology, Inc.), anti-TATA-binding protein (TBP) $(1: 2,000$; cat. no. ab818; Abcam) and anti- $\beta$-actin (1:5,000; cat. no. A1978; Sigma-Aldrich; Merck KgaA). The blots were then incubated with goat anti-rabbit horse-radish peroxidase (HRP)-conjugated secondary antibodies (1:5,000; cat. no. ADI-SAB-300-J; Enzo Life Sciences, Inc.) or goat anti-mouse HRP-conjugated secondary antibodies (1:5,000; cat. no. ADI-SAB-100-J; Enzo Life Sciences, Inc.) at room temperature for $1.5 \mathrm{~h}$ and then developed using Immobilon ${ }^{\circledR}$ Western Chemiluminescent HRP substrate (Merck KgaA).

RNA isolation and RT-PCR. HUVECs were treated with $20 \mu \mathrm{M}$ CA or MCA for various periods of time. Total RNA was isolated using TRI-solution (Bio Science Technology) according to the manufacturer's instructions. Semi-quantitative RT-PCR was performed using an ONE-STEP RT-PCR PreMix kit (iNtRON Biotechnology) according to the manufacturer's instructions. The specific primers and cycling conditions for RT-PCR have been previously described (15). The RT-PCR products were electrophoresed on $1.7 \%$ agarose gels and visualized by ethidium bromide staining.

Detection of ROS. HUVECs were treated with $10 \mu \mathrm{M}$ ZnPP. After $2 \mathrm{~h}$, the cells were treated with $20 \mu \mathrm{M}$ CA for $24 \mathrm{~h}$ and then further treated with $350 \mu \mathrm{M} \mathrm{H}_{2} \mathrm{O}_{2}$ for an additional $6 \mathrm{~h}$. The levels of generated ROS were measured by staining the cells with $10 \mu \mathrm{M}$ dihydroethidium for $30 \mathrm{~min}$ according to a previously described method (15). Fluorescence was analyzed using a conventional fluorescence microscope (Axio Observer D1, Carl Zeiss AG).

Transfection with siRNA. HUVECs were seeded in a 12-well plate at a density of $7 \times 10^{4}$ cells $/ \mathrm{ml}$, cultured overnight and transfected with either $25 \mathrm{nM}$ of HO-1 siRNA (5'-GGCAGA GAAUGCUGAGUUC-3') or 5 nM of Nrf2 siRNA (sc-37030, Santa Cruz Biotechnology, Inc.) using DharmaFECT transfection reagent (GE Healthcare Dharmacon, Inc.) according to the manufacturer's instructions. After $6 \mathrm{~h}$, the media were 
changed, and the cells were further incubated for $18 \mathrm{~h}$ before being treated with $20 \mu \mathrm{M}$ CA. siGENOME Non-Targeting siRNA Pool (GE Healthcare Dharmacon, Inc.) was used as a control.

Annexin $V$ apoptosis assay. HUVECs were treated with $20 \mu \mathrm{M} \mathrm{CA}$ or $5 \mu \mathrm{M}$ CoPP for $24 \mathrm{~h}$ and then with $350 \mu \mathrm{M}$ $\mathrm{H}_{2} \mathrm{O}_{2}$ for $24 \mathrm{~h}$. An Annexin $\mathrm{V}$ apoptosis assay was performed with a Muse ${ }^{\mathrm{TM}}$ Annexin V \& Dead Cell kit according to the manufacturer's protocol (Merck KGaA). Briefly, $100 \mu \mathrm{l}$ of a cell suspension was mixed with $100 \mu \mathrm{l}$ of Muse ${ }^{\mathrm{TM}}$ Annexin V and Dead Cell reagent and then incubated at room temperature for $20 \mathrm{~min}$. Apoptotic cells were analyzed using a Muse Cell Analyzer (Merck KGaA).

U937 cell adhesion assay. U937 cells were labeled with calcein AM fluorescent dye $(1 \mu \mathrm{g} / \mathrm{ml})$ for $30 \mathrm{~min}$ at $37^{\circ} \mathrm{C}$. The cells were then washed with PBS and resuspended in ECM. HUVECs were cultured in a $35 \mathrm{~mm}$ dish until confluent, after which the cells were treated with CA $(20 \mu \mathrm{M})$ and/or TNF- $\alpha$ (10 $\mathrm{ng} / \mathrm{ml})$ for $6 \mathrm{~h}$. The HUVECs were then co-cultured with calcein AM-labeled U937 cells for $3 \mathrm{~h}$. Non-adhering U937 cells were removed by washing twice with PBS. Finally, the HUVECs were fixed with $4 \%$ paraformaldehyde in PBS for $10 \mathrm{~min}$ and then mounted using CRYSTAL/MOUNT ${ }^{\mathrm{TM}}$ mounting medium (Biomeda Corp.). Fluorescence analysis was performed using a conventional fluorescence microscope.

Animals, lipopolysaccharide (LPS) administration and histological analysis. A total of 25 male Sprague-Dawley rats (4 weeks old, weighing 80-100 g, Samtaco Bio) were used in the present study. The animals were housed in a controlled environment with a temperature of $24 \pm 1^{\circ} \mathrm{C}$ and a humidity of $50 \pm 5 \%$ with a $12 \mathrm{~h}$ light and dark cycle. Food and water were provided ad libitum. The rats $(\mathrm{n}=5$ in each group) were intraperitoneally injected with CA $(50 \mathrm{mg} / \mathrm{kg})$. After $3 \mathrm{~h}$, LPS $(0.5 \mathrm{mg} / \mathrm{kg})$ was inoculated subcutaneously into the right flanks of the rats. After $24 \mathrm{~h}$, the rats were sacrificed, subcutaneous tissues were excised, and then fixed in $10 \%$ buffered formalin and embedded in paraffin. Tissue sections (4- $\mu$ m-thick) were stained with Hematoxylin (Sigma-Aldrich; Merck KgaA) for 5 min and eosine (Sigma-Aldrich; Merck KgaA) for $10 \mathrm{sec}$ at RT and then examination by light microscopy.

Ethics statement. Animal care procedures were approved by the Chosun University Institutional Animal Care and Use Committee (CIACUC2020-A0012). All research was conducted in accordance with the provided protocol.

Statistical analysis. The data are expressed as the means \pm SD of 3 independent experiments. Statistical analysis was performed using SPSS 24 (IBM, Corp.). Differences between groups were analyzed by ANOVA followed by Tukey's post hoc test. A P-value $<0.05$ was considered to indicate a statistically significant difference.

\section{Results}

Effect of CA and its derivatives on HUVEC viability and cytotoxicity. To evaluate CA and its natural derivatives, MCA and
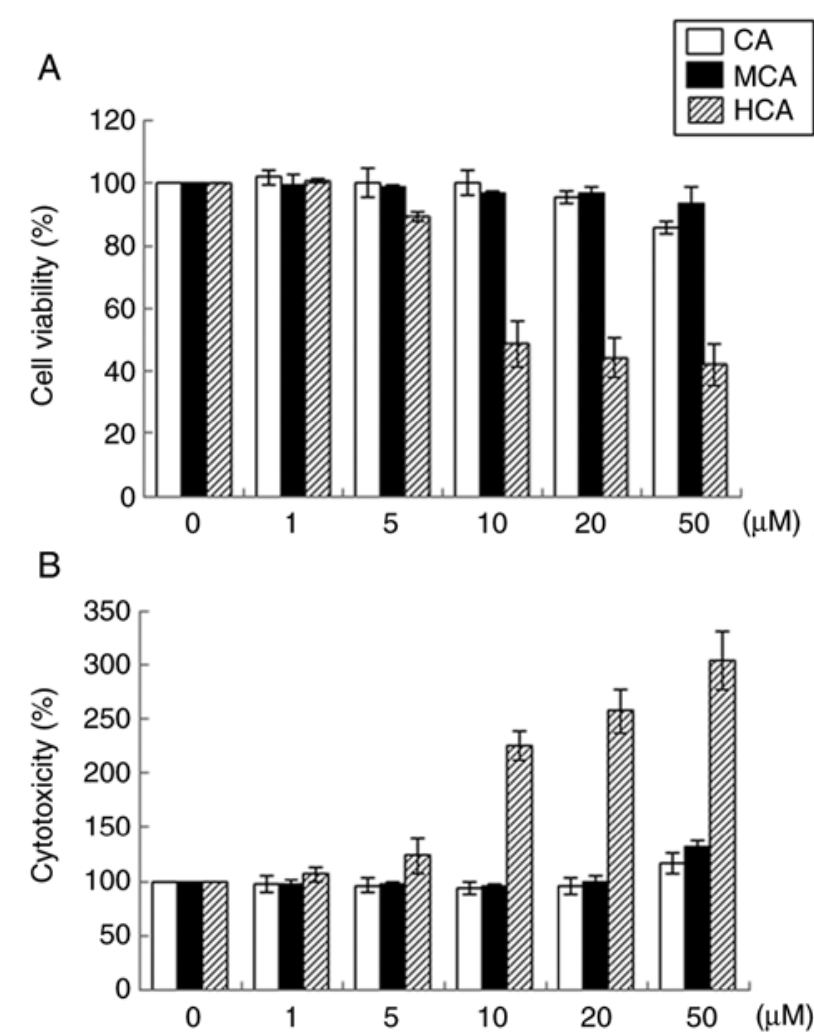

Figure 1. Effects of CA and its derivatives on cell viability and cytotoxicity. HUVECs were treated with the indicated concentrations of CA, MCA or HCA for $24 \mathrm{~h}$. (A) Cell viability was assessed by the MTT assay. (B) Cell cytotoxicity was measured by the LDH assay. The data are expressed as means \pm SD of 3 separate experiments. HUVECs, human umbilical vein endothelial cells; CA, cinnamaldehyde; MCA, 2-methoxycinnamaldehyde; HCA, 2'-hydroxycinnamaldehyde.

HCA, as possible candidates for the treatment of atherosclerosis, their effects on HUVEC viability were examined. HCA markedly decreased cell viability in a dose-dependent manner (Fig. 1A). Cells treated with $50 \mu \mathrm{M}$ HCA exhibited $57.7 \%$ lower viability compared with the untreated control HUVECs. HCA also markedly induced cytotoxicity in a dose-dependent manner (Fig. 1B). However, CA and MCA at concentrations of up to $50 \mu \mathrm{M}$ did not induce any notable changes in cell viability or cytotoxicity. Therefore, $\mathrm{CA}$ and MCA were used in the subsequent experiments.

$C A$ and MCA induce the expression of HO-1. Recently, it was reported that CA induces HO-1 expression in hDPCs (15). Therefore, the present study examined the effects of $\mathrm{CA}$ and MCA on HO-1 expression in HUVECs. As shown in Fig. 2A and B, both CA and MCA induced the expression of HO-1 in a dose- and time-dependent manner. By showing that CA and MCA increased the mRNA level of HO-1 (Fig. 2C), it was confirmed that $\mathrm{CA}$ and $\mathrm{MCA}$ regulate $\mathrm{HO}-1$ at the transcriptional level.

CA protects cells from oxidative stress by inducing HO-1 expression. Previous studies have demonstrated that HO-1 exerts cytoprotective effects against oxidative stress $(22,23)$. Therefore, the cytoprotective effects of CA and MCA on HUVECs were evaluated in the present 
A

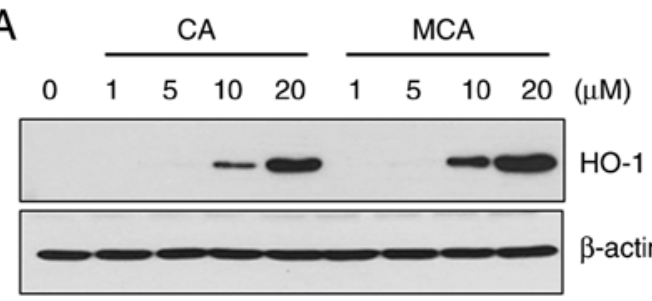

$\mathrm{C}$
B
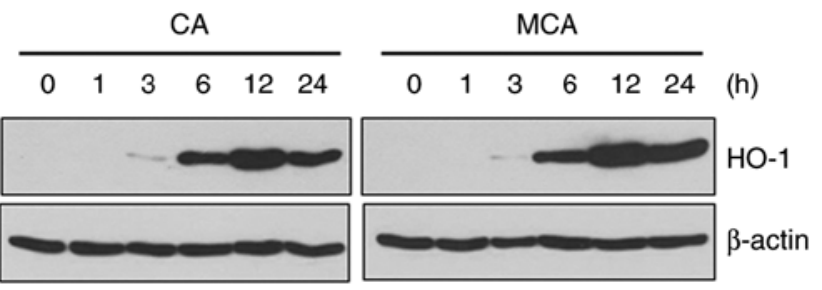

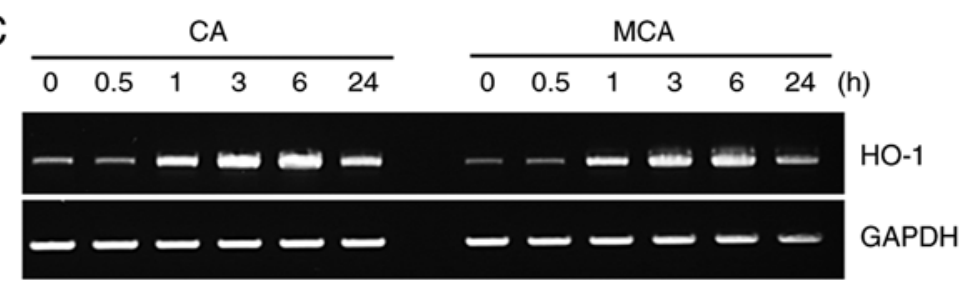

Figure 2. CA and MCA induce the expression of HO-1. (A) HUVECs were treated with the indicated concentrations of CA or MCA for 24 h. Total cell lysates were prepared, and the levels of HO-1 were detected by western blot analysis. (B and C) Cells were treated with $20 \mu \mathrm{M} \mathrm{CA}$ or MCA for the indicated periods of time. Total cell lysates were prepared, and the levels of HO-1 were detected by western blot analysis (B). (C) Alternatively, total RNA was isolated, and levels of HO-1 mRNA were detected by semi-quantitative RT-PCR. CA, cinnamaldehyde; MCA, 2-methoxycinnamaldehyde; HCA, 2'-hydroxycinnamaldehyde; HO-1, heme oxygenase-1.
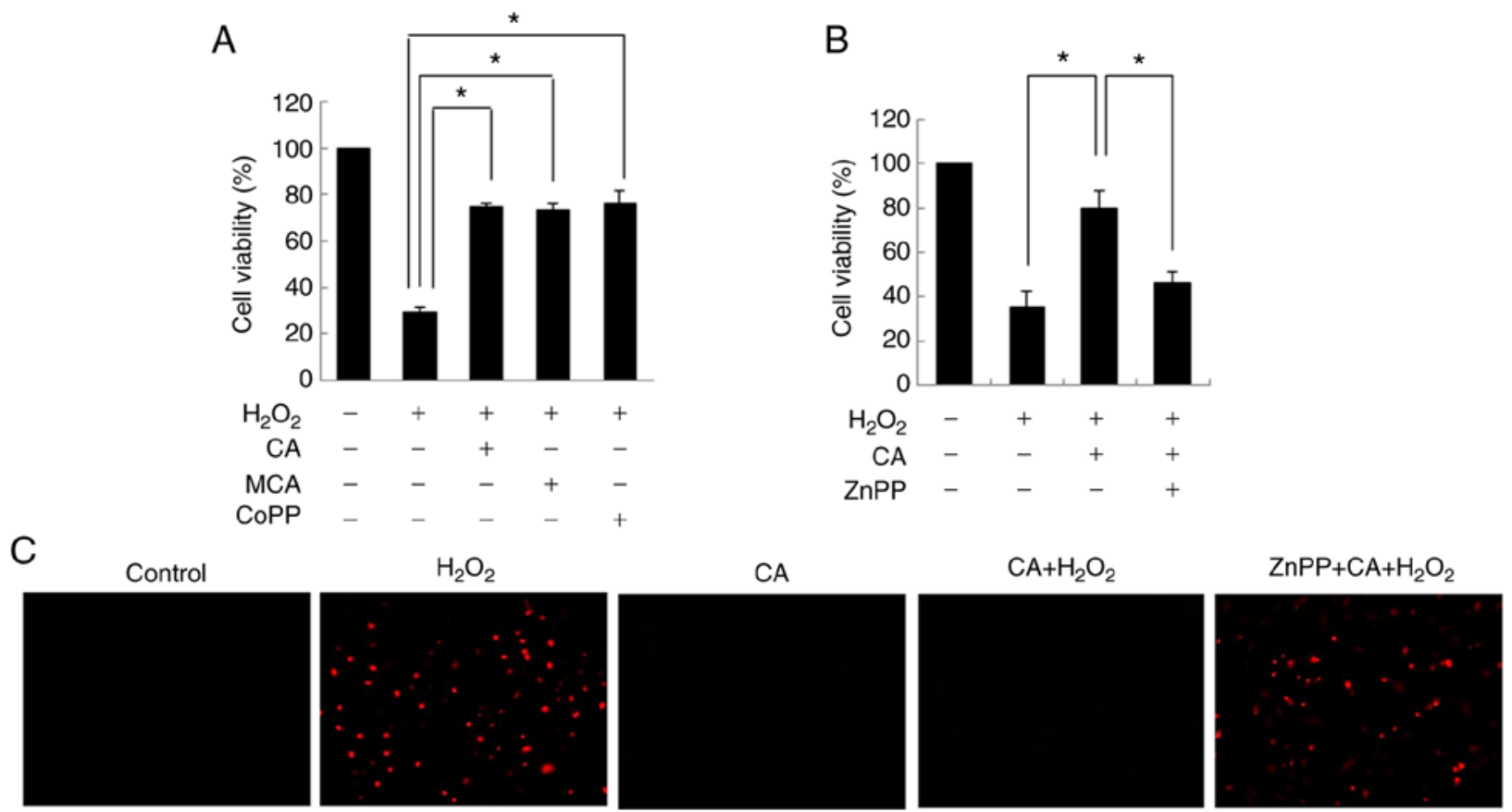

Figure 3. CA protects cells from $\mathrm{H}_{2} \mathrm{O}_{2}$-induced oxidative stress by inducing HO-1 expression. (A) HUVECs were pre-treated with $20 \mu \mathrm{M}$ CA or MCA, or $5 \mu \mathrm{M}$ CoPP for $24 \mathrm{~h}$. The cells were then further treated with $\mathrm{H}_{2} \mathrm{O}_{2}$ for $24 \mathrm{~h}$, and cell viability was assessed by MTT assay. (B) HUVECs were pre-treated with $10 \mu \mathrm{M} \mathrm{ZnPP}$ for $2 \mathrm{~h}$ and then treated with CA for $24 \mathrm{~h}$. The cells were further treated with $\mathrm{H}_{2} \mathrm{O}_{2}$ for an additional $24 \mathrm{~h}$, and an MTT assay was performed to assess cell viability. "P<0.05. (C) Cells were pre-treated with $\mathrm{ZnPP}$ for $2 \mathrm{~h}$ and then with CA for $24 \mathrm{~h}$ and $\mathrm{H}_{2} \mathrm{O}_{2}$ for a further $6 \mathrm{~h}$. After the cells were stained with dihydroethidium, the level of generated ROS was monitored by fluorescence microscopy. HUVECs, human umbilical vein endothelial cells; CA, cinnamaldehyde; MCA, 2-methoxycinnamaldehyde; CoPP, Cobalt protoporphyrin; ZnPP, zinc protoporphyrin.

study. Exposure to $\mathrm{H}_{2} \mathrm{O}_{2}$ decreased cell viability. However, pre-treatment with both CA and MCA inhibited $\mathrm{H}_{2} \mathrm{O}_{2}$-induced cell cytotoxicity (Fig. 3A). CoPP, an HO-1 inducer, exerted an inhibitory effect similar to that of CA and MCA, suggesting that the cytoprotective effects of CA and MCA are mainly mediated via the induction of $\mathrm{HO}-1$.

To confirm the role of HO-1 in $\mathrm{H}_{2} \mathrm{O}_{2}$-induced cytotoxicity, HUVECs were treated with ZnPP, an inhibitor of HO-1. As shown in Fig. 3B, the cytoprotective effects of CA were almost completely inhibited by ZnPP pre-treatment. ROS staining also demonstrated that CA effectively reduced the $\mathrm{H}_{2} \mathrm{O}_{2}$-induced intracellular ROS levels (Fig. 3C), and ZnPP completely reversed the inhibitory effects of CA on ROS generation induced by $\mathrm{H}_{2} \mathrm{O}_{2}$, confirming that $\mathrm{HO}-1$ plays a role in the CA-mediated cytoprotective effect on HUVECs.

The cytoprotective effects of $\mathrm{CA}$ against $\mathrm{H}_{2} \mathrm{O}_{2}$-induced apoptosis was also evaluated by flow cytometry. $\mathrm{H}_{2} \mathrm{O}_{2}$ markedly increased apoptotic cell death compared with the untreated HUVECs (Fig. 4A). However, CA effectively inhibited $\mathrm{H}_{2} \mathrm{O}_{2}$-induced apoptosis. Although pre-treatment with 

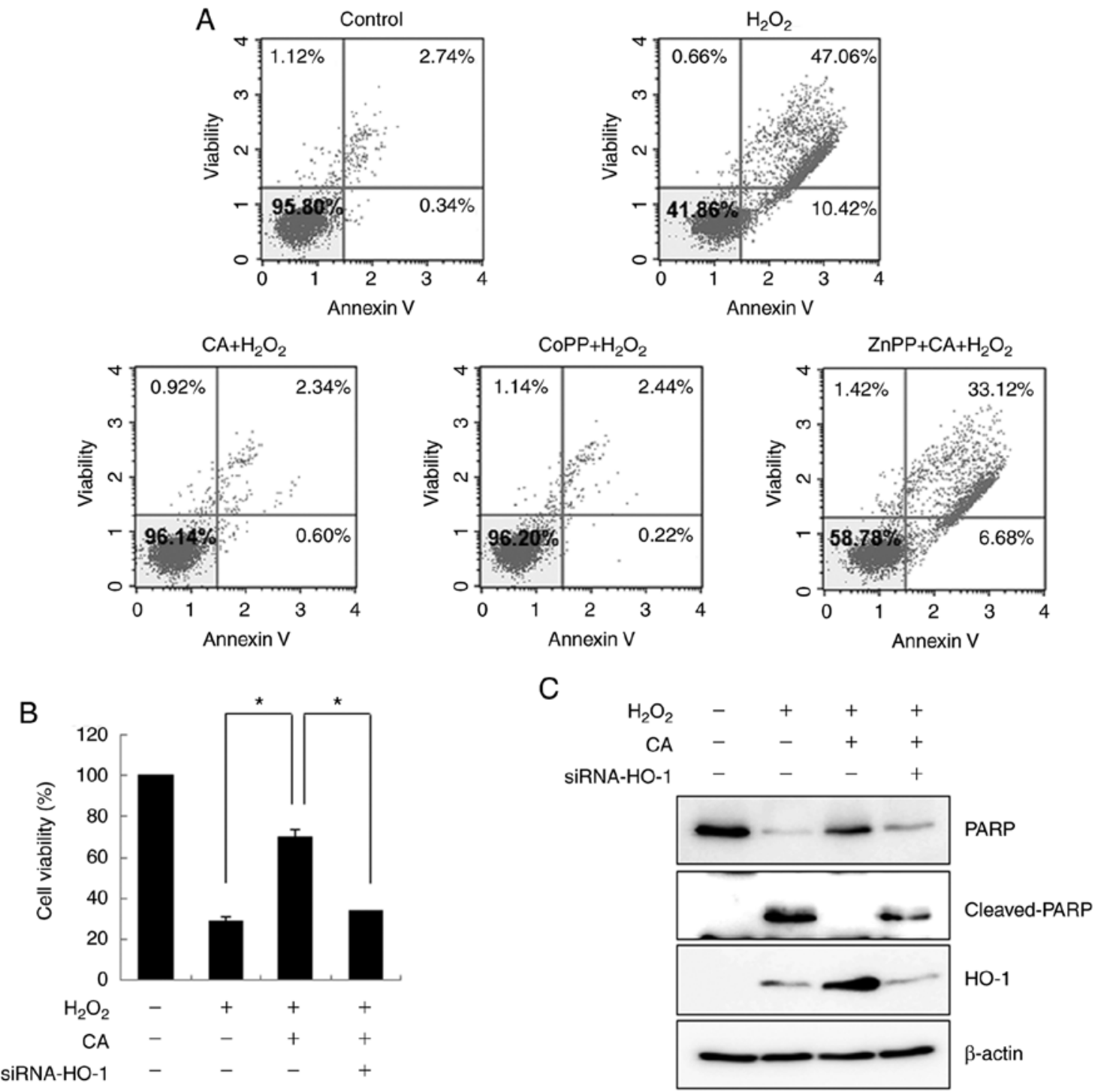

Figure 4. HO-1 is actively involved in CA-mediated cytoprotective effects against $\mathrm{H}_{2} \mathrm{O}_{2}$-induced apoptosis. (A) Cells were treated with $\mathrm{CA}$ for $24 \mathrm{~h}$ and then treated with $\mathrm{H}_{2} \mathrm{O}_{2}$ for a further $24 \mathrm{~h}$. Apoptotic cells were analyzed by a Muse ${ }^{\mathrm{TM}}$ Annexin V \& Dead Cell kit using a Muse ${ }^{\mathrm{TM}}$ Cell Analyzer. (B and C) Cells were transfected with siRNA against HO-1. After $6 \mathrm{~h}$, the media were changed, and the cells were incubated for an additional $6 \mathrm{~h}$. The cells were then treated with $\mathrm{CA}$ for $24 \mathrm{~h}$ and $\mathrm{H}_{2} \mathrm{O}_{2}$ for a further $12 \mathrm{~h}$. Cell viability was determined by MTT assay. The data are expressed as the means $\pm \mathrm{SD}$ of 3 individual experiments. "P<0.05. (C) Total cell lysates were prepared, and the level of cleaved-PARP was determined by western blot analysis. CA, cinnamaldehyde; MCA, 2-methoxycinnamaldehyde; CoPP, Cobalt protoporphyrin; ZnPP, zinc protoporphyrin; HO-1, heme oxygenase-1; PARP, poly(ADP-ribose) polymerase.

ZnPP failed to protect the cells from oxidative stress, CoPP exerted a cytoprotective effect to an extent similar to that of $\mathrm{CA}$, revealing that $\mathrm{HO}-1$ plays an important role in oxidative stress-induced cell death.

To verify the role of $\mathrm{HO}-1$ in $\mathrm{H}_{2} \mathrm{O}_{2}$-induced cell death, HUVECs were transfected with $\mathrm{HO}-1$-specific siRNA. As depicted in Fig. 4B, transfection with HO-1-siRNA abrogated the cytoprotective effects of $\mathrm{CA}$ on $\mathrm{H}_{2} \mathrm{O}_{2}$-induced apoptosis. By demonstrating that $\mathrm{CA}$ treatment decreased the level of cleaved PARP and that this cytoprotective effect was abolished by HO-1-siRNA, the role of HO-1 in CA-mediated cytoprotection was confirmed (Fig. 4C). The transfection efficiency of the siRNA was confirmed by demonstrating that the induced level of HO-1 was effectively inhibited by HO-1-siRNA.

CA induces HO-1 expression through the p38-mediated Nrf2 signaling pathway. To determine the signaling mechanisms responsible for CA-induced HO-1 expression, western blot analysis was performed. CA markedly induced the nuclear translocation of Nrf2, although it did not affect $\mathrm{NF}-\kappa \mathrm{B}$ nuclear translocation, as illustrated in Fig. 5A. As Nrf2-specific siRNA inhibited HO-1 expression in a dose-dependent manner, $\mathrm{CA}$ was confirmed to induce $\mathrm{HO}-1$ expression through $\mathrm{Nrf} 2$ activation (Fig. 5B).

The involvement of MAPKs in CA-induced Nrf2 activation was then examined. CA did not affect the phosphorylation levels of AKT and ERK; however, the phosphorylated levels of p38 were markedly increased (Fig. 5C). JNK was not detected under the same experimental conditions (data not shown). Treatment with SB202190, an inhibitor of p38, decreased HO-1 expression in a dose-dependent manner, suggesting the involvement of the p38 signaling pathway in CA-induced HO-1 expression (Fig. 5D). Furthermore, treatment with SB202190 inhibited the nuclear translocation of Nrf2 (Fig. 5E), 
A

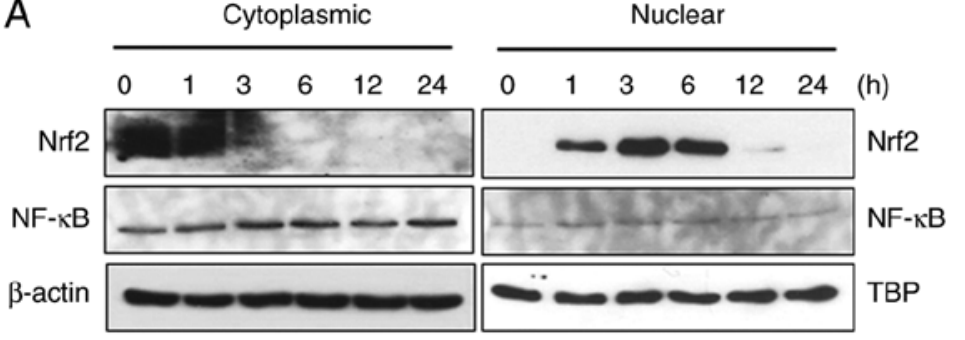

$\mathrm{B} \quad \mathrm{CA}-++++$

siRNA-Nrf2 $\quad-\quad-\begin{array}{llll}1 & 2 & 5 & (\mu \mathrm{M})\end{array}$

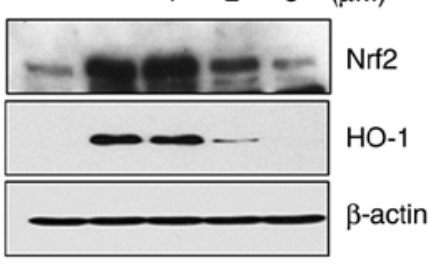

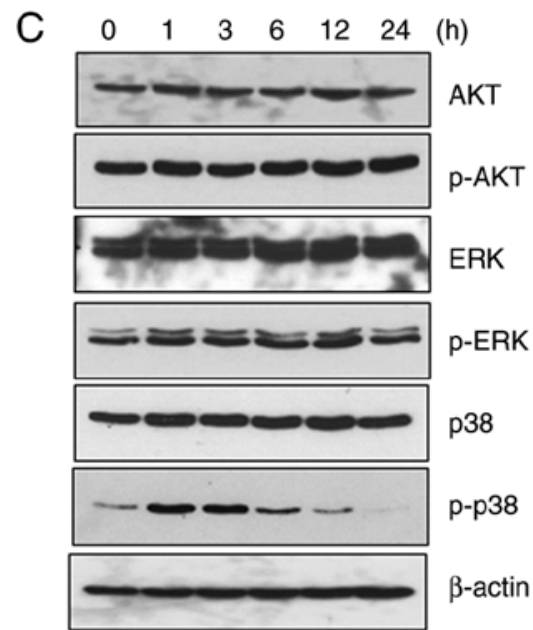

D

$\mathrm{CA}-+++++$ SB202190 - $\quad-\begin{array}{lllll}5 & 10 & 20 & 50 & (\mu \mathrm{M})\end{array}$

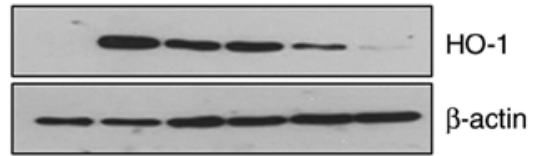

E

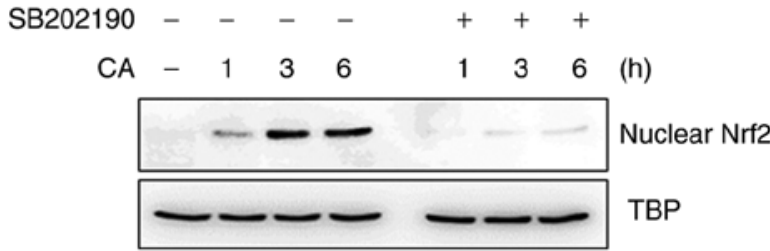

Figure 5. CA induces HO-1 expression through $\mathrm{p} 38$ and subsequent Nrf2 activation. (A) HUVECs were treated with CA for the indicated periods of time. Cytoplasmic and nuclear fractions were prepared, and the levels of Nrf2 and NF- $\mathrm{kB}$ were detected by western blot analysis. (B) Cells were transfected with siRNA against Nrf2. After $6 \mathrm{~h}$, the media were changed, and the cells were incubated for an additional $18 \mathrm{~h}$. The cells were then treated with CA for $24 \mathrm{~h}$. Total cell lysates were prepared, and levels of Nrf2 and HO-1 were detected by western blot analysis. (C) Cells were treated with CA for the indicated periods of time. Total cell extracts were prepared and subjected to western blot analysis. (D) Cells were pre-treated with SB202190 for $2 \mathrm{~h}$ and then with CA for an additional $24 \mathrm{~h}$. Total cell extracts were prepared, and the levels of HO-1 were detected by western blot analysis. (E) Cells were pre-treated with SB202190 for $2 \mathrm{~h}$ and then with CA for the indicated periods of time. Nuclear fractions were prepared, and the levels of Nrf2 were detected by western blot analysis. CA, cinnamaldehyde; $\mathrm{Nrf} 2$, nuclear factor erythroid 2-related factor 2; HO-1, heme oxygenase-1.

confirming the involvement of the $\mathrm{p} 38$ signaling pathway in CA-induced Nrf2/HO-1 activation.

CA inhibits U937 cell adhesion to HUVECs. To further evaluate CA as a candidate anti-atherosclerotic agent, a U937 cell adhesion assay with HUVECs was performed. As shown in Fig. 6A, TNF- $\alpha$-induced U937 cell adhesion was markedly inhibited by $\mathrm{CA}$ treatment.

The adhesion of monocytes/macrophages to the endothelium is induced by adhesion molecules, such as ICAM-1 and VCAM-1. Therefore, the expression levels of these adhesion molecules in CA-treated HUVECs were examined.CA treatment induced a dose-dependent decrease in VCAM-1 expression, whereas it has no effect on ICAM-1 expression (Fig. 6B). MCA exerts effects similar to those of CA. As the expression of HO-1 and VCAM-1 was inversely regulated by CA, the association between HO-1 and VCAM-1 expression was then assessed. As shown in Fig. 6C, the level of HO-1 did not affect the level of VCAM-1, suggesting that CA independently regulates the expression levels of HO-1 and VCAM-1. The transfection efficiency of HO-1-siRNA was confirmed by examining the level of HO-1. NF- $\mathrm{NB}$ is a well-known transcription factor that modulates inflammatory cytokines and adhesion molecules. Although CA did not affect the phosphorylation of NF- $\kappa \mathrm{B}$ induced by TNF- $\alpha$, it effectively inhibited the TNF- $\alpha$-induced nuclear translocation of NF- $\kappa \mathrm{B}$ (Fig. 6D).
CA inhibits inflammatory cell infiltration. To examine the anti-inflammatory effects of CA in vivo, Sprague-Dawley rats were pre-treated with CA and then exposed to LPS, as described in the Materials and methods section. Various concentrations (20 mg to $100 \mathrm{mg} / \mathrm{kg}$ ) of CA have been applied to tumor and inflammatory animal models $(24,25)$. Based on previous studies, the present study examined the anti-inflammatory effects of CA (20 and $50 \mathrm{mg} / \mathrm{kg}$ ) in an animal model. In a preliminary experiment, the $50 \mathrm{mg} / \mathrm{kg}$ treated group exhibited a more effective anti-inflammatory effect than the $20 \mathrm{mg} / \mathrm{kg}$-treated group (data not shown). Therefore, CA $50 \mathrm{mg} / \mathrm{kg}$ was used in the in vivo experiments. After $24 \mathrm{~h}$, subcutaneous tissues were subjected to histological examination. LPS induced excessive inflammatory cell infiltration in subcutaneous tissues (Fig. 7). However, CA pre-treatment decreased the LPS-induced inflammatory cell infiltration, verifying the anti-inflammatory effects of CA. Treatment with CA alone did not affect subcutaneous tissues.

\section{Discussion}

ROS are oxygen-containing compounds that are highly reactive with biomolecules, such as lipids, proteins and DNA. $\mathrm{H}_{2} \mathrm{O}_{2}$ is a key ROS, along with $\mathrm{O}_{2}^{-}$and ${ }^{\circ} \mathrm{OH}$. Endogenous $\mathrm{H}_{2} \mathrm{O}_{2}$ is generated through the respiratory chain and as a byproduct of cellular metabolism. Exogenous $\mathrm{H}_{2} \mathrm{O}_{2}$ can also enter cells 
A
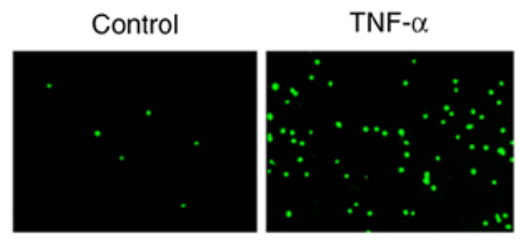

B

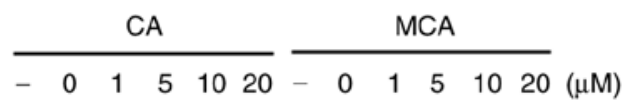

TNF- $\alpha$

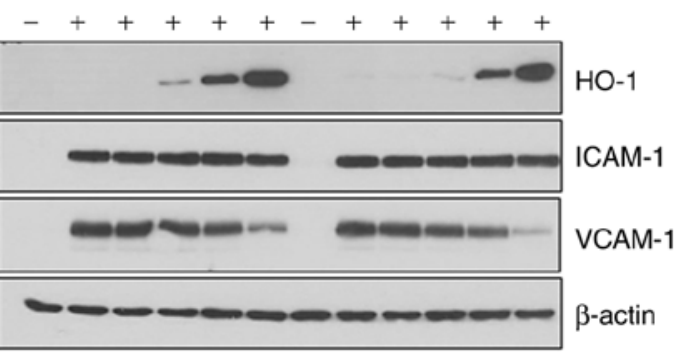

TNF- $\alpha+C A$

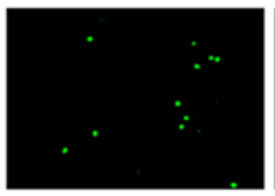

C
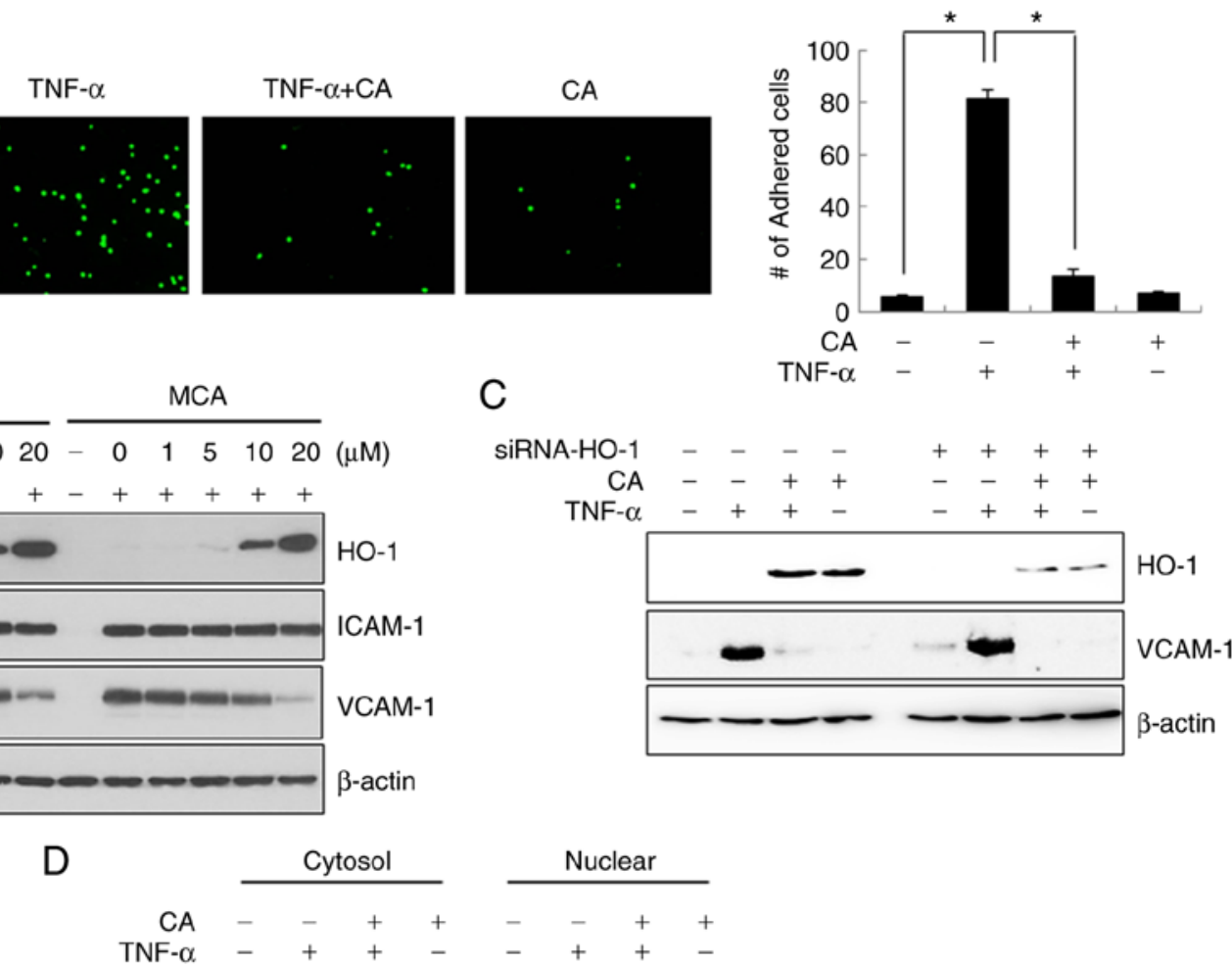

Nuclear
-++

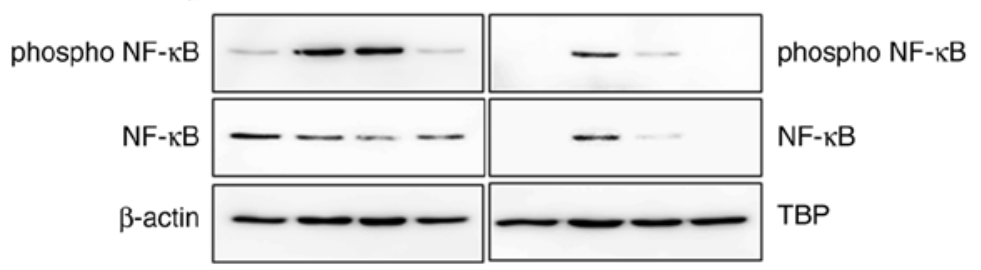

Figure 6. CA inhibits TNF- $\alpha$-induced U937 cell adhesion. (A) HUVECs were treated with TNF- $\alpha$ and/or CA for 3 h. The cells were then co-cultured with calcein-AM-labeled U937 cells for $3 \mathrm{~h}$, after which they were fixed with paraformaldehyde and analyzed by fluorescence microscopy. The data are expressed as the means \pm SD of 3 individual experiments. ${ }^{*} \mathrm{P}<0.05$. (B) HUVECs were treated with TNF- $\alpha$ and/or various concentrations of CA or MCA for $24 \mathrm{~h}$. Total cell lysates were prepared, and the levels of HO-1, ICAM-1 and VCAM-1 were detected by western blot analysis. (C) Cells were transfected with siRNA against HO-1. After $6 \mathrm{~h}$, the media were changed, and the cells were incubated for an additional $6 \mathrm{~h}$. The cells were then treated with TNF- $\alpha$ and/or CA for $24 \mathrm{~h}$. Total cell lysates were prepared, and western blot analysis was performed. (D) Cells were treated with TNF- $\alpha$ and/or CA for 24 h. Cytoplasmic and nuclear frac-

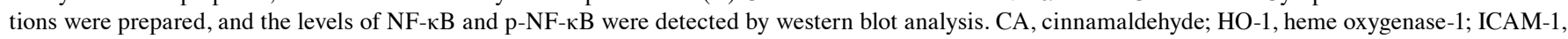
intercellular adhesion molecule 1; VCAM-1, vascular cell adhesion molecule 1; TNF- $\alpha$, tumor necrosis factor $\alpha$.
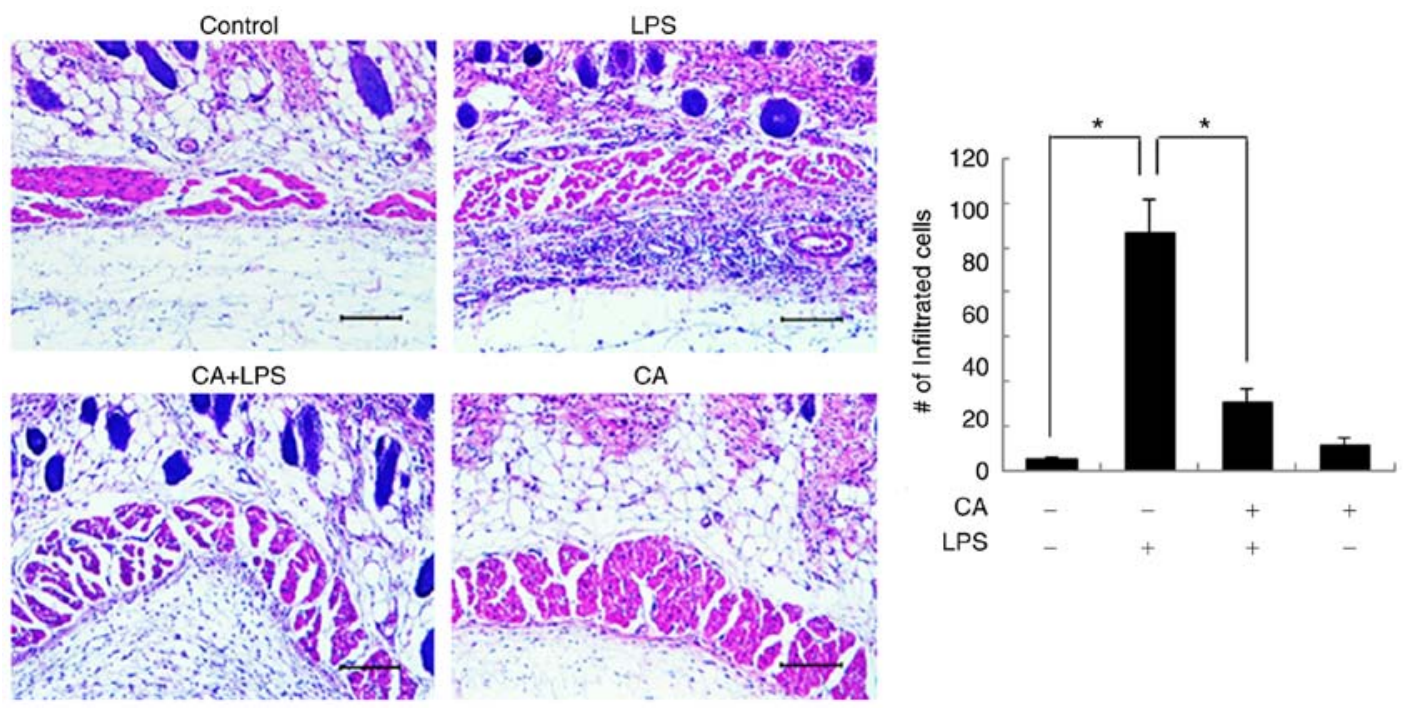

Figure 7. CA inhibits inflammatory cell infiltration in vivo. Rats were intraperitoneally injected with CA. After $3 \mathrm{~h}$, LPS was subcutaneously inoculated into the right flanks of the rats. After $24 \mathrm{~h}$, subcutaneous tissues were excised and stained with H\&E. Inflammatory cell infiltration was evaluated by light microscopy. Original magnification, $\mathrm{x} 100$; scale bar, $10 \mu \mathrm{m}$. The graph shows the quantitative evaluation of the infiltrated cells. The data are expressed as the means $\pm \mathrm{SD}$ of 3 individual experiments. "P<0.05. CA, cinnamaldehyde; LPS, lipopolysaccharide. 
through simple diffusion or facilitated diffusion by aquaporins $(26,27)$. As ROS are an important risk factor for the development of cardiovascular disease, antioxidant therapies that scavenge ROS are considered an effective strategy for anti-atherosclerotic treatment $(5,6)$.

Vascular endothelial cells play a critical role in the maintenance of vascular homeostasis, and endothelial dysfunction is involved in the pathogenesis of diverse cardiovascular and inflammatory diseases (28). To the best of our knowledge, the present study evaluated, for the first time, the antioxidant activity of CA and its derivatives in HUVECs. Stem bark-derived cinnamon is composed of $45-65 \%$ CA (7). HCA and MCA, natural derivatives of CA, contain hydroxy and methoxy groups, respectively, at the CA 2' site (19). The authors of the present study, as well as others have demonstrated that HCA exhibits potent anti-tumor activity in various types of cancer cells by inducing cell cycle arrest and subsequent apoptosis (29-31). Consistent with these studies, the data of the present study clearly demonstrated that HCA inhibited cell viability and induced cytotoxicity (Fig. 1). Therefore, HCA was ruled out as a candidate anti-atherosclerotic agent.

Recently, it was demonstrated that CA exhibits antioxidant activity by enhancing the expression of HO-1 through the Nrf2 signaling pathway in hDPCs (15). The present study verified that both CA and MCA induced the expression of HO-1 and protected HUVECs against oxidative stress through an HO-1-mediated mechanism. It was also demonstrated that CA activated Nrf2 nuclear translocation and subsequent HO-1 expression. Nrf2 plays a critical role in protecting cells from various toxic insults, such as oxidative stress. Under normal conditions, Nrf2 is inactive and localized in the cytosol. Upon cellular insult, activated Nrf2 is translocated to the nucleus, where it binds antioxidant response elements (AREs) to activate target gene expression $(16,17)$. Several signaling pathways, such as the AKT and ERK pathways, have been reported to activate the Nrf2/HO-1 pathway (32). In the present study, it was demonstrated that CA induced the phosphorylation of p38 and that a specific inhibitor of p38, SB202190, completely abolished not only Nrf2 nuclear translocation, but also subsequent HO-1 expression (Fig. 5). These data suggest that the induction of the p38 pathway by CA induces Nrf2 nuclear translocation, leading to HO-1 expression. However, it was previously reported by the authors that $\mathrm{CA}$-induced activation of Nrf2/HO-1 signaling in hDPCs does not involve MAPK signaling pathways (15). Furthermore, Huang et al demonstrated that CA activated the ERK, AKT and JNK pathways, but not the p38 pathway in HepG2 hepatoma cells (33). Therefore, the activation of Nrf2 by CA appears to be controversial and cell-type specific.

Inflammation is also a critical factor in the development of atherogenic processes $(12,34)$. TNF- $\alpha$, a well-known major inflammatory cytokine that induces inflammatory responses in the vascular endothelium, has been identified in atherosclerotic plaque specimens (35). TNF- $\alpha$ stimulates endothelial cell adhesion to circulating monocytes by inducing the expression of the cell adhesion molecules ICAM-1 and VCAM-1. The present study demonstrated that both CA and MCA effectively reduced the expression of VCAM-1 and led to the inhibition of U937 monocytic cell adhesion to endothelial cells (Fig. 6).
Nonetheless, siRNA specific for HO-1 did not affect the level of VCAM-1, suggesting that the anti-inflammatory effect of CA was not related to HO-1 expression. Consistent with these results, $\mathrm{CA}$ inhibited the TNF- $\alpha$-induced nuclear translocation of NF- $\mathrm{KB}$.

In the present study, it was found that CA effectively protected HUVECs from $\mathrm{H}_{2} \mathrm{O}_{2}$-induced oxidative stress through the activation of the Nrf2 signaling pathway and the subsequent induction of HO-1. The anti-inflammatory effects of CA were also verified by demonstrating that CA inhibits monocyte adhesion to endothelial cells by decreasing the expression of VCAM-1. Taken together, these findings strongly suggest that CA and MCA are possible candidates for atherosclerosis treatment.

\section{Acknowledgements}

Not applicable.

\section{Funding}

The present study was supported by the National Research Foundation of Korea (NRF) grant funded by the Korea government (MEST) (grant no. NRF-2017R1A2B4009239).

\section{Availability of data and materials}

All data generated or analyzed during this study are available from the corresponding author on reasonable request.

\section{Authors' contributions}

NYK and NTT performed the experiments and analyzed the data. SGA designed, analyzed and performed the animal experiment. SAK designed the study, analyzed the data and wrote the manuscript. All authors read and approved the final manuscript.

\section{Ethics approval and consent to participate}

Animal care procedures were approved by the Chosun University Institutional Animal Care and Use Committee (CIACUC2020-A0012). All research was conducted in accordance with the provided protocol.

\section{Patient consent for publication}

Not applicable.

\section{Competing interests}

The authors declare that they have no competing interests.

\section{References}

1. Apel K and Hirt H: Reactive oxygen species: Metabolism, oxidative stress, and signal transduction. Annu Rev Plant Biol 55: 373-399, 2004.

2. D'Autreaux B and Toledano MB: ROS as signaling molecules: Mechanisms that generate specificity in ROS homeostasis. Nat Rev Mol Cell Biol 8: 813-824, 2007. 
3. Kim SM, Hwang KA and Choi KC: Potential roles of reactive oxygen species derived from chemical substances involved in cancer development in the female reproductive system. BMB Rep 51: 557-562, 2018.

4. Pisoschi AM and Pop A: The role of antioxidants in the chemistry of oxidative stress: A review. Eur J Med Chem 97: 55-74, 2015.

5. Ochoa CD, Wu RF and Terada LS: ROS signaling and ER stress in cardiovascular disease. Mol Aspects Med 63: 18-29, 2018.

6. Pignatelli P, Menichelli D, Pastori D and Violi F: Oxidative stress and cardiovascular disease: New insights. Kardiol Pol 76 713-722, 2018

7. Shen Y, Jia LN, Honma N, Hosono T, Ariga T and Seki T: Beneficial effects of cinnamon on the metabolic syndrome, inflammation, and pain, and mechanisms underlying these effects-a review. J Tradit Complement Med 2: 27-32, 2012.

8. Ahn SG, Jin YH, Yoon JH and Kim SA: The anticancer mechanism of 2'-hydroxycinnamaldehyde in human head and neck cancer cells. Int J Oncol 47: 1793-1800, 2015.

9. Chao LK, Hua KF, Hsu HY, Cheng SS, Lin IF, Chen CJ, Chen ST and Chang ST: Cinnamaldehyde inhibits pro-inflammatory cytokines secretion from monocytes/macrophages through suppression of intracellular signaling. Food Chem Toxicol 46 : 220-231, 2008

10. Kwon JA, Yu CB and Park HD: Bacteriocidal effects and inhibition of cell separation of cinnamic aldehyde on Bacillus cereus Lett Appl Microbiol 37: 61-65, 2003.

11. Song F, Li H, Sun J and Wang S: Protective effects of cinnamic acid and cinnamic aldehyde on isoproterenol-induced acute myocardial ischemia in rats. J Ethnopharmacol 150: 125-130, 2013.

12. Fan $J$ and Watanabe $T$ : Inflammatory reactions in the pathogenesis of atherosclerosis. J Atheroscler Thromb 10: 63-71, 2003.

13. Incalza MA, D'Oria R, Natalicchio A, Perrini S, Laviola L and Giorgino F: Oxidative stress and reactive oxygen species in endothelial dysfunction associated with cardiovascular and metabolic diseases. Vascul Pharmacol 100: 1-19, 2018.

14. Kim SM, Huh JW, Kim EY, Shin MK, Park JE, Kim SW, Lee W, Choi B and Chang EJ: Endothelial dysfunction induces atherosclerosis: Increased aggrecan expression promotes apoptosis in vascular smooth muscle cells. BMB Rep 52: 145-150, 2019.

15. Kim NY, Ahn SG and Kim SA: Cinnamaldehyde protects human dental pulp cells against oxidative stress through the Nrf2/HO-1-dependent antioxidant response. Eur J Pharmacol 815 : 73-79, 2017

16. Chen XL and Kunsch C: Induction of cytoprotective genes through Nrf2/antioxidant response element pathway: A new therapeutic approach for the treatment of inflammatory diseases. Curr Pharm Des 10: 879-891, 2004

17. Niture SK, Khatri R and Jaiswal AK: Regulation of Nrf2-an update. Free Radic Biol Med 66: 36-44, 2014.

18. Li W, Zhi W, Zhao J, Yao Q, Liu F and Niu X: Cinnamaldehyde protects VSMCs against ox-LDL-induced proliferation and migration through $\mathrm{S}$ arrest and inhibition of p38, JNK/MAPKs and NF-кB. Vascul Pharmacol 108: 57-66, 2018.

19. Jin YH and Kim SA: 2-methoxycinnamaldehyde inhibits the TNF- $\alpha$-induced proliferation and migration of human aortic smooth muscle cells. Int J Mol Med 39: 191-198, 2017.

20. Kim SA, Kim YC, Kim SW, Lee SH, Min JJ, Ahn SG and Yoon JH: Antitumor activity of novel indirubin derivatives in rat tumor model. Clin Cancer Res 13: 253-259, 2007.
21. Jin YH, Ahn SG and Kim SA: BAG3 affects the nucleocytoplasmic shuttling of HSF1 upon heat stress. Biochem Biophys Res Commun 464: 561-567, 2015.

22. Mo L, Yang C, Gu M, Zheng D, Lin L, Wang X, Lan A, Hu F and Feng J: PI3K/Akt signaling pathway-induced heme oxygenase-1 upregulation mediates the adaptive cytoprotection of hydrogen peroxide preconditioning against oxidative injury in PC12 cells. Int J Mol Med 30: 314-320, 2012

23. Takahashi T, Morita K, Akagi R and Sassa S: Heme oxygenase-1: A novel therapeutic target in oxidative tissue injuries. Curr Med Chem 11: 1545-1561, 2004.

24. Chiang YF, Chen HY, Huang KC, Lin PH and Hsia SM: Dietary antioxidant trans-cinnamaldehyde reduced visfatin-induced breast cancer progression: In vivo and in vitro study. Antioxidants (Basel) 8: E625, 2019.

25. Patra K, Jana S, Sarkar A, Mandal DP and Bhattacharjee S: The inhibition of hypoxia-induced angiogenesis and metastasis by cinnamaldehyde is mediated by decreasing HIF- $1 \alpha$ protein synthesis via PI3K/Akt pathway. Biofactors 45: 401-415, 2019.

26. Holmstrom KM and Finkel T: Cellular mechanisms and physiological consequences of redox-dependent signalling. Nat Rev Mol Cell Biol 15: 411-421, 2014.

27. Schieber $M$ and Chandel NS: ROS function in redox signaling and oxidative stress. Curr Biol 24: R453-R462, 2014.

28. Li B, Lee YJ, Kim YC, Yoon JJ, Lee SM, Lee YP, Kang DG and Lee HS: Sauchinone from saururus chinensis protects vascular inflammation by heme oxygenase-1 induction in human umbilical vein endothelial cells. Phytomedicine 21: 101-108, 2014.

29. Lee CW, Lee SH, Lee JW, Ban JO, Lee SY, Yoo HS, Jung JK, Moon DC, Oh KW and Hong JT: 2-hydroxycinnamaldehyde inhibits SW620 colon cancer coll growth through AP-1 inactivation. J Pharmacol Sci 104: 19-28, 2007.

30. Kim SA, Sung YK, Kwon BM, Yoon JH, Lee H, Ahn SG and Hong SH: 2'-hydroxycinnamaldehyde shows antitumor activity against oral cancer in vitro and in vivo in a rat tumor model. Anticancer Res 30: 489-494, 2010.

31. Nguyen HA and Kim SA: 2'-hydroxycinnamaldehyde induces apoptosis through HSF1-mediated BAG3 expression. Int J Oncol 50: 283-289, 2017.

32. Luo Y, Lu S, Dong X, Xu L, Sun G and Sun X: Dihydromyricetin protects human umbilical vein endothelial cells from injury through ERK and Akt mediated Nrf2/HO-1 signaling pathway. Apoptosis 22: 1013-1024, 2017.

33. Huang TC, Chung YL, Wu ML and Chuang SM: Cinnamaldehyde enhances Nrf2 nuclear translocation to upregulate phase II detoxifying enzyme expression in HepG2 cells. J Agric Food Chem 59: 5164-5171, 2011.

34. Kim EJ, Park WH, Ahn SG, Yoon JH, Kim SW and Kim SA: 5'-nitro-indirubinoxime inhibits inflammatory response in TNF-alpha stimulated human umbilical vein endothelial cells. Atherosclerosis 211: 77-83, 2010.

35. Barath P, Fishbein MC, Cao J, Berenson J, Helfant RH and Forrester JS: Detection and localization of tumor necrosis factor in human atheroma. Am J Cardiol 65: 297-302, 1990.

This work is licensed under a Creative Commons Attribution-NonCommercial-NoDerivatives 4.0 International (CC BY-NC-ND 4.0) License. 